$\mathrm{O}$ ver 1.5 million percutaneous coronary revascularisation procedures are performed annually world wide, most being intracoronary stenting. Despite enormous advances in devices, the major limitation is in-stent stenosis (ISS) (reviewed by Bennett and $\mathrm{O}^{\prime}$ Sullivan $^{1}$ ). Although ISS rates are $10-20 \%$ in selected patients, we now stent total occlusions, saphenous vein bypass grafts, both angioplasty and ISS sites, diabetic patients, and small vessels. Thus, real ISS rates are much higher, up to 59\% in some high risk lesions.

Until recently, the only effective treatment for ISS was brachytherapy. Brachytherapy reduces target vessel revascularisation rates and binary restenosis rates, and increases minimum luminal diameters (MLDs) compared with control vessels, maintained to three years.' Although effective, brachytherapy is not universally accepted, due predominantly to late thrombosis and the logistics of administering radioactivity. In contrast, drug eluting stents containing the immunosuppressive agent rapamycin and the antimitotic agent paclitaxel have shown encouraging reductions in ISS in de novo lesions, ${ }^{2}{ }^{3}$ and possibly in ISS lesions. This review examines the pathology of ISS and how drugs bound to stents interrupt the normal response to vessel injury.

\title{
MECHANISMS OF RESTENOSIS
}

Overdistension of the diseased vessel causes endothelial disruption, internal elastic lamina fracture, and medial dissection. Lumen enlargement is caused by a combination of plaque reduction (compression/embolisation), axial plaque redistribution towards the proximal and distal segments outside the stent, plaque extrusion, and vessel expansion. Many processes then contribute to restenosis (fig 1 and table 1 ).

\section{Elastic recoil}

The human epicardial coronary arteries contain elastin fibres comprising the internal and external elastic laminae (IEL/EEL). Elastic recoil therefore follows overstretch, occurring between seconds and minutes following balloon deflation, resulting in up to $40 \%$ loss of luminal areas. Although both angioplasty and stenting produce overstretch injury, stenting significantly reduces acute recoil.

\section{Reorganisation of thrombus}

Angioplasty or stenting causes endothelial denudation and induces medial dissection. The consequent exposure of subintimal components such as collagen, Von Willebrand factor, fibronectin, and laminin causes platelet adherence and aggregation. Fibrin and platelets are deposited on stent struts early after implantation. The association of fibrin and platelets with neointimal accumulation and extensive neovascularisation at ISS sites suggests that organisation of mural thrombus promotes ISS.

\section{Neointimal formation}

Arterial injury induces vascular smooth muscle cell (VSMC) proliferation and/or migration due to: (1) mechanical stretch, IEL rupture, and medial dissection; (2) endothelial denudation with exposure to circulating mitogens such as angiotensin II and plasmin; and (3) release of mitogens and cytokines from platelets, endothelial cells, VSMCs, and inflammatory cells. VSMCs are normally in the quiescent $\left(G_{0}\right)$ phase of the cell cycle. In animal models, injury induces medial and then intimal cell cycle entry, following a wave of immediate early gene expression. Cells undergo either cell proliferation, migration or both, with subsequent synthesis of extracellular matrix and collagen, resulting in neointima formation.

Neointimal formation is the major cause of ISS. Neointima increases up to three months after Professor Martin R Bennett, Addenbrooke's Centre for Clinical Investigation, Box 110, Addenbrooke's Hospital, Cambridge CB2 2QQ, UK; mrb@mole.bio.cam.ac.uk the procedure, with little change to six months, and a gradual reduction between six months and three years. However, the role of cell proliferation within the neointima remains controversial. Cell proliferation is low in specimens retrieved from ISS sites, although higher in ISS versus angioplasty restenosis sites. ${ }^{4}$ Although neointima formation after stenting is associated with medial disruption 
A

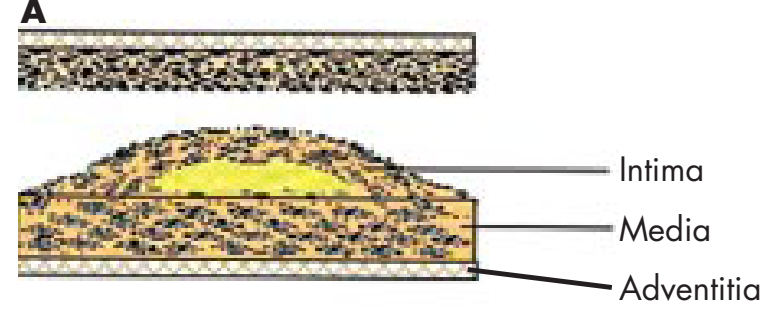

B

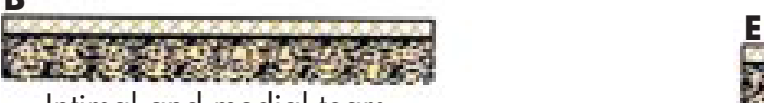

Intimal and medial tears
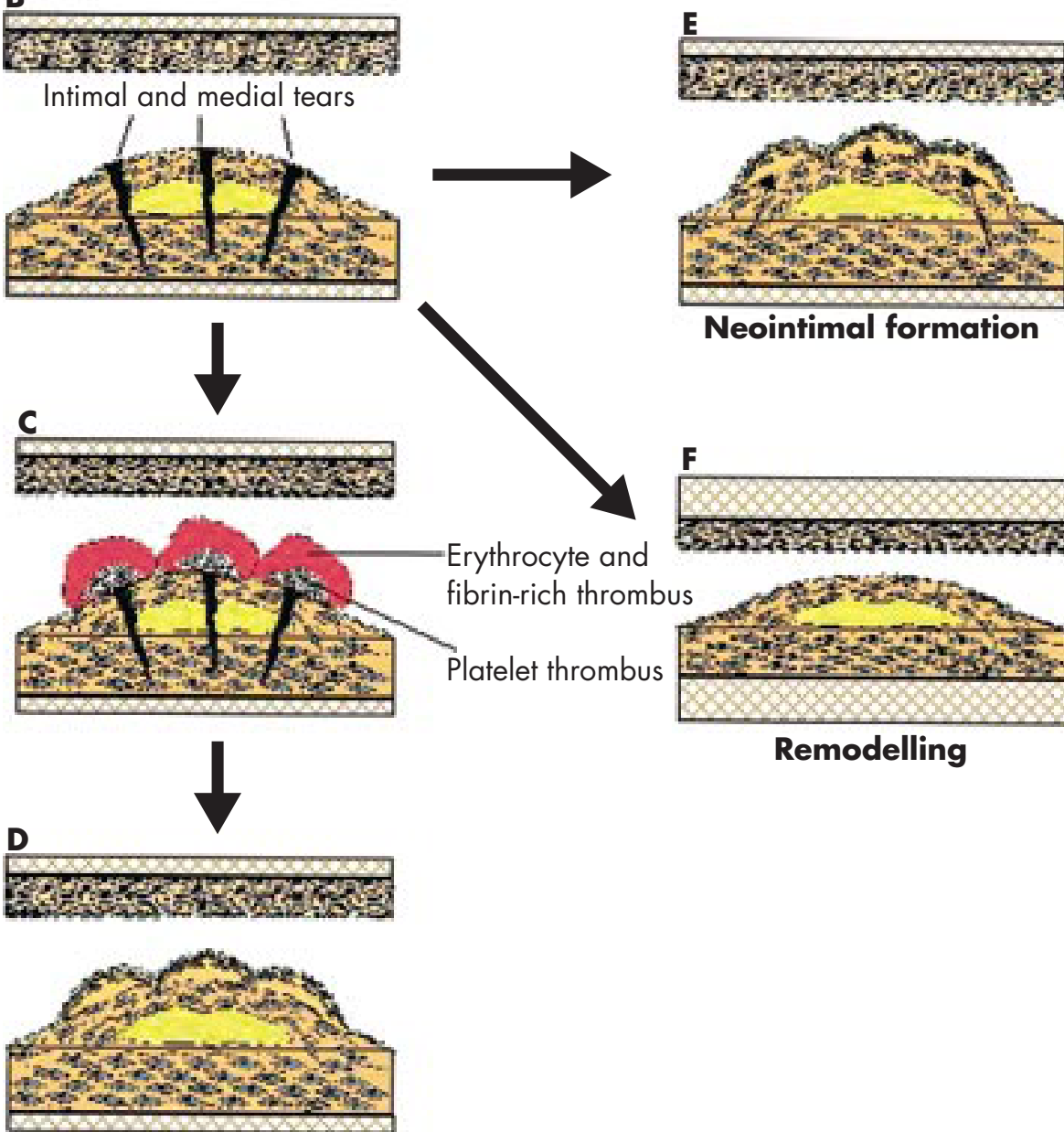

Neointimal formation

F
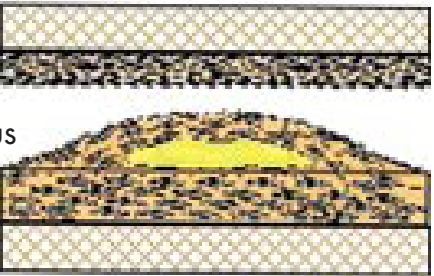

Remodelling
Figure 1 Possible outcomes following angioplasty and mechanisms responsible for restenosis. (A) Schematic representation of the diseased vessel before intervention, showing the structure of the vessel wall and a stenotic vessel segment. (B) Following angioplasty there is dissection of both the intima of the plaque and the vessel media, associated with an enlargement of the treated segment. Angioplasty is either successful acutely, or may be complicated by acute thrombosis and vessel occlusion. Early failure is also caused by elastic recoil following dilation. Late failure may be caused by the formation of both platelet and fibrin/erythrocyte-rich thrombi (C) that then get remodelled by vascular smooth muscle cells (VSMCs) invading from the media or intima to form a neointima (D). Neointima formation may also result from migration and proliferation of medial and intimal VSMCs following injury (E). In contrast, restenosis may be caused by negative remodelling (F). Here, the injury to both the media and adventitia results in cell proliferation and collagen synthesis, expanding the adventitia. Subsequent maturation of collagen is associated with contraction and overall vessel shrinkage. It is important to note that an increase in neointimal mass may not necessarily cause restenosis if sufficient (positive) remodelling of the vessel occurs, and negative remodelling may result in restenosis with minimal increase in neointimal mass. Intracoronary stents function predominantly to inhibit elastic recoil and negative remodelling. Reprinted from Bennett M R, O'Sullivan M. Pharmacology and Therapeutics 2001;91:149-66, with permission from Elsevier Science.

\section{Reorganisation of thrombus}

(extent of injury), ${ }^{5}$ proliferation does not correlate with time of injury. In contrast, most proliferating cells are located deep, adjacent to stent struts, ${ }^{6}$ suggesting that proliferation is a chronic low grade reaction to the stent.

Importantly, the restenotic lesion after angioplasty or stenting is hypocellular compared with primary plaques, consisting of collagen and matrix proteoglycans produced by limited VSMCs. Indeed, cells comprise only $11 \%$ of the tissue mass in human post-angioplasty neointima. ${ }^{7}$ ISS also consists of matrix proteoglycans and collagen, with decreasing cellularity and increasing matrix content as ISS develops. This lack of VSMC proliferation in ISS clearly may reduce the expected benefit of treatments aimed solely at cell proliferation, compared with those that additionally alter matrix synthesis.
"Neointima" formation within a stent also results from other mechanisms, such as axial movement of primary plaque displaced to adjacent artery segments by the original procedure. Although this may represent VSMC "migration", true VSMC migration from the media or adventitia adjacent to the angioplasty site analogous to that seen in animal models has not been conclusively demonstrated in humans.

\section{Remodelling}

Negative remodelling is a major cause of human angioplasty restenosis, where $>40 \%$ of specimens retrieved at necropsy show no evidence of neointimal formation. ${ }^{8}$ Intravascular ultrasound studies also show that remodelling causes between two thirds and three quarters of the lumen loss in restenosis lesions. ${ }^{9}$ Although remodelling is largely negated by stenting, 
Table 1 Pathogenic processes leading to in-stent stenosis

\begin{aligned} & \hline Early (within days) Elastic recoil \\ & Late (weeks to months) Relocation of axially transmitted plaque \\ & Reorganisation of thrombus \\ & Neointima formation \\ & -cell proliferation \\ & -cell migration \\ & -cell matrix synthesis \\ & Remodelling \\ & Resolution of inflammation \\ & \hline\end{aligned}

the mechanisms that contribute to remodelling are unknown, or whether remodelling represents primarily a medial or adventitial response to injury.

\section{Resolution of inflammation}

Porcine and non-primate models of injury show a robust inflammatory reaction to injury, with early mononuclear cell infiltration from the lumen into the thrombus. Monocytes secrete fibrinolytic enzymes, which may remodel or resorb the thrombus. Human stented arteries also show acute inflammation early after implantation, especially when stenting is associated with medial injury or lipid core penetration, ${ }^{5}{ }^{10}$ and restenosis is associated with core penetration and inflammation. Indeed, some inflammatory cells (predominantly macrophages) are found at all stages of stent stenosis. ${ }^{10}$ As described above, resolution of inflammation may therefore play an important part in restenosis, in particular via fibrosis and resultant scar contraction of both the adventitia and media.

\section{TREATMENT AND PREVENTION OF IN-STENT STENOSIS}

From the above discussion, it is clear that multiple biological processes promote ISS, each involving a plethora of molecules. This strongly argues for the use of agents or treatments that are not specific to one molecular target, unless that target signals in a variety of biological processes. Bearing this in mind, it is informative to examine how the most successful treatments, brachytherapy and drug eluting stents, utilise these properties.

\section{Brachytherapy}

Intracoronary brachytherapy, using either $\beta$ or $\gamma$ sources, has emerged as a promising new modality for the prevention or treatment of in-stent restenosis. Brachytherapy reduces neointimal accumulation, probably by blocking cell proliferation, inducing apoptosis, and inhibiting cell migration. Brachytherapy also prevents constrictive remodelling, probably by reducing the healing response with subsequent fibrosis.

Patients with in-stent restenosis treated with brachytherapy have lower target vessel revascularisation rates, lower binary restenosis rates, and larger MLDs than control vessels, up to three years after the procedure (summarised in table 2). The effects of brachytherapy are evident at six months, but persist to three years. Brachytherapy appears to target both neointimal formation, with reductions in cell proliferation noted in both media and adventitia, and a reduction in adventitial myofibroblast accumulation; irradiation also results in improved (often positive) geometric remodelling, either by effects on medial remodelling or adventitial fibrosis, and this luminal enlargement is also seen in humans.

Unfortunately brachytherapy also has significant deleterious effects, including late thrombosis resulting in significantly higher rates of late myocardial infarction, failure of medial dissections to heal, paradoxical stenosis at the ends or outside of the stents, acceleration of neointimal thickening at some doses or with chronic therapy, and failure of endothelial regeneration (table 3). Problems with late (to nine months) thrombosis and myocardial infarction mainly occur when antiplatelet agents are stopped, and/or patients receive new stents at time of irradiation; however, concerns over myocardial infarction at later time points still persist, based in part on evidence of incomplete healing and recurrent thrombus formation after brachytherapy in animal models. Edge restenosis is a particular problem with radioactive stents, and brachytherapy also poses significant logistic difficulties related to the administration of radiation outside of clinical oncology. Thus, a more desirable approach to the problem would be a drug with the broad spectrum acute effects of brachytherapy, but without the profound effects of delayed healing.

Although brachytherapy may not be the final solution to ISS, the non-specificity of the treatment is both its strength, targeting multiple processes in restenosis, and its weakness, resulting in failure to heal. In contrast, drug eluting stents using a carefully selected group of drugs at the correct dose and administration seem to offer the benefits without the side effects of brachytherapy. The most promising of these are rapamycin (sirolimus) and paclitaxel.

\section{Drug eluting stents}

Rapamycin

Rapamycin (sirolimus) possesses weak antibiotic activity, but is a potent modulator of immune function, acting both on $\mathrm{T}$

Table 2 Summary of landmark brachytherapy trials

\begin{tabular}{|c|c|c|c|c|c|c|c|}
\hline & BERT & SCRIPPS & GAMMA- WRIST & BETA-WRIST & PREVENT & GAMMA-ONE & $\begin{array}{l}\beta \text { Irradiation: the } \\
\text { Dose-Finding } \\
\text { Study Group }\end{array}$ \\
\hline Trial design & Radiation & $\begin{array}{l}\text { Radiation } v \\
\text { placebo }\end{array}$ & $\begin{array}{l}\text { Radiation } v \\
\text { placebo }\end{array}$ & Radiation & $\begin{array}{l}\text { Radiation } v \\
\text { placebo }\end{array}$ & $\begin{array}{l}\text { Radiation } v \\
\text { placebo }\end{array}$ & Radiation \\
\hline Type of radiation & $\begin{array}{l}\beta \text { (strontium/ } \\
\text { yttrium 90) }\end{array}$ & $\gamma$ (iridium 192) & $\gamma$ (iridium 192) & $\beta($ yttrium-90) & $\beta$ (phosphorus 32) & $\gamma$ (iridium 192) & $\beta$ (yttrium-90) \\
\hline Number of patients & 21 & 55 & 130 & 50 & 105 & 252 & 181 \\
\hline Lesion treated & PTCA sites & $\begin{array}{l}\text { Restenosis after } \\
\text { PTCA or stenting }\end{array}$ & In-stent stenosis & In-stent stenosis & $\begin{array}{l}\text { De novo or } \\
\text { restenosis lesions }\end{array}$ & In-stent stenosis & $\begin{array}{l}\text { Restenosis after } \\
\text { PTCA or stenting }\end{array}$ \\
\hline $\begin{array}{l}\text { Restenosis rates ( } 6 \\
\text { months) }\end{array}$ & $15 \%$ & $17 \%$ v $54 \%$ & $19 \%$ v $58 \%$ & $22 \%$ & $22 \%$ v $50 \%$ & $32.4 \%$ v $55.3 \%$ & $\begin{array}{l}15 \% \text { (for highest } \\
\text { dose) }\end{array}$ \\
\hline TVR at 6 months & $15 \%$ & $12 \% \vee 45 \%$ (TLR) & $26.2 \%$ v $67.7 \%$ & $34 \%$ & $\begin{array}{l}6 \% \text { v } 24 \%(21 \% v \\
32 \% \text { at } 12 \text { months) }\end{array}$ & $31.3 \%$ v $46.3 \%$ & $\begin{array}{l}6 \% \text { (for highest } \\
\text { dose) }\end{array}$ \\
\hline
\end{tabular}




\begin{tabular}{|lll|}
\hline \multicolumn{2}{|l|}{ Table 3 Pathological/clinical sequelae of brachytherapy/drug eluting stents } \\
\hline Response & Manifested by & Clinical sequelae \\
\hline $\begin{array}{l}\text { Injury without treatment } \\
\text { (geographical miss) }\end{array}$ & $\begin{array}{c}\text { Acceleration of neointimal thickening at some } \\
\text { doses or with chronic treatment }\end{array}$ & $\begin{array}{l}\text { Edge restenosis } \\
\text { In-stent stenosis }\end{array}$ \\
Failure of healing & Lack of endothelialisation & Late thrombosis \\
& Fibrin deposition/intraintimal haemorrhage & Late thrombosis \\
& Intimal inflammation & ? Negative remodelling \\
& Adventitial inflammation & ? In-stent stenosis \\
& Failure of medial dissections to heal & ? Negative remodelling \\
& Vascular smooth muscle cell death & Late thrombosis \\
& & ? Negative remodelling \\
\hline
\end{tabular}

lymphocyte activation and cell proliferation. The mammalian target of rapamycin (mTOR) is a large evolutionarily conserved member of the phosphatidylinositol kinase (PIK) related kinase family that regulates protein translation, cell cycle progression, and cell proliferation. MTOR is a critical and essential regulator of many second messenger pathways within eukaryotic cells such that TOR inactivation is developmentally lethal. Rapamycin enters cells easily where it is bound to a specific intracellular receptor $\mathrm{FKBP}_{12}$-the rapamycin/FKBP ${ }_{12}$ complex is a highly specific inhibitor of mTOR.

Growth factor activation of cells activates the kinase activity of mTOR, using a number of classical intracellular signalling systems, including PI-3 kinase and the serine threonine kinase Akt (fig 2). mTOR, together with PI-3K dependent signals phosphorylates downstream effectors, the best characterised of which are p70 S6 kinase (S6K1) and 4EBP-1. S6 kinase directly phosphorylates the $40 \mathrm{~S}$ ribosomal protein S6, which correlates with increased translation of sequences that encode components of the translational machinery. Phosphorylation of the translational repressor 4EBP-1 ultimately leads to increased cap dependent translation through eukaryotic initiation factor (eiF) 4E-F. Thus, rapamycin inhibits protein synthesis. S6 kinase also phosphorylates many other downstream proteins, such as CBP80 (functions in RNA export and splicing), eEF2 kinase (protein translation), and BAD (apoptosis), potentially controlling a variety of cellular processes. Growth factor activation of cells also downregulates the cyclin dependent kinase inhibitor (cdki) p27, in addition to increasing expressing of cyclins, predominantly cyclin D and E; these events are required for

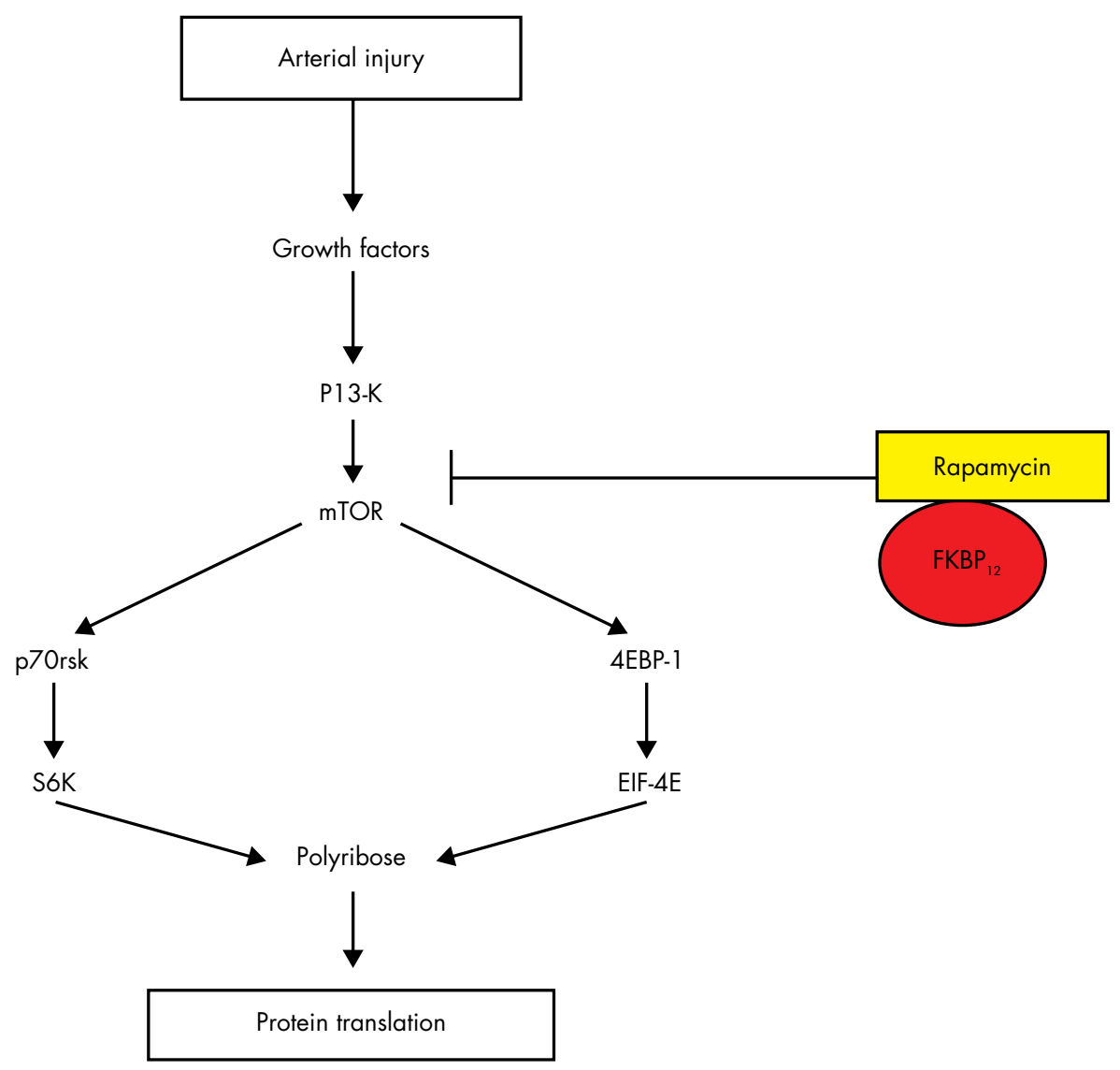

Figure 2 The effect of rapamycin is mediated via binding to a specific intracellular receptor, FKBP $_{12}$, with subsequent inactivation of the enzyme mTOR, and reduced activity of mTOR targets including S6 kinase and 4EP-1. For explanation of abbreviations see text. 


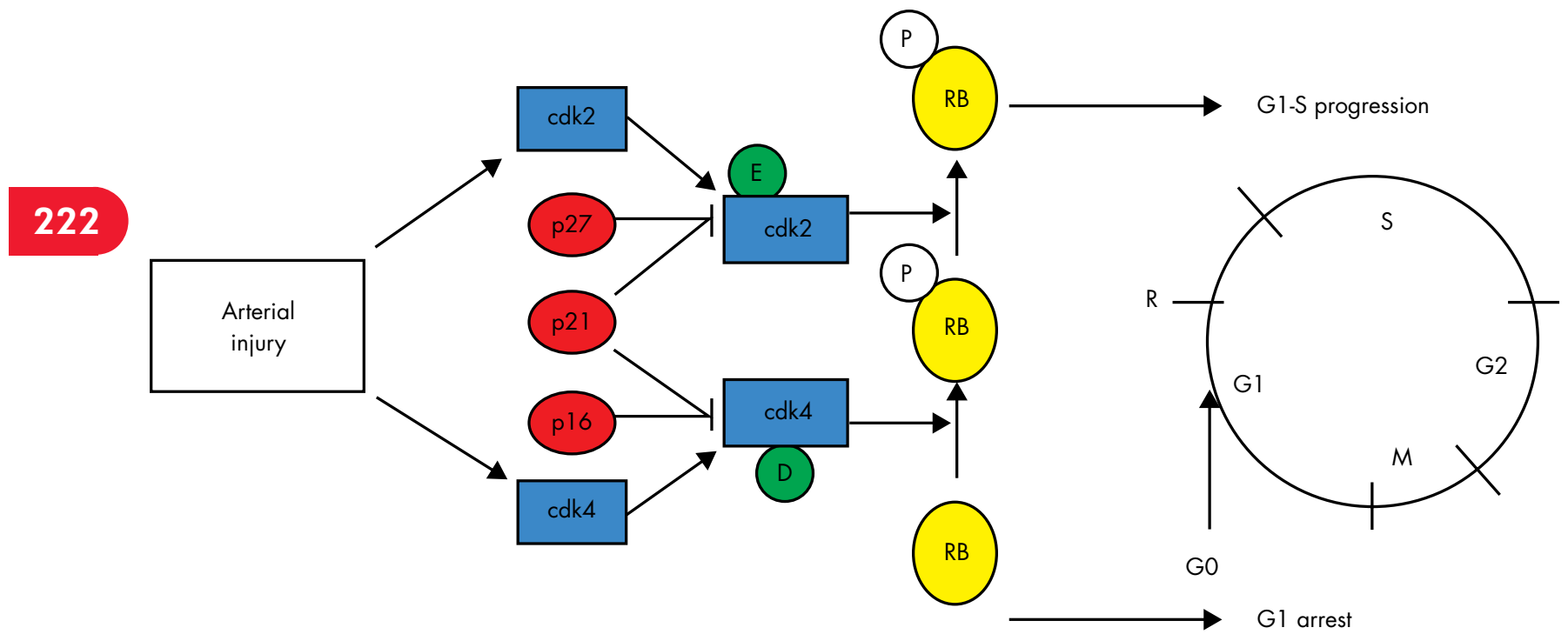

Figure 3 Artery injury induces growth factor release, resulting in increased expression of cdks 2 and 4, downregulation of p27, with resultant increased activity of cdk2/cyclin E and cdk4/cyclin D complexes. These enzymes phosphorylate (inactivate) pRB allowing passage of cells from $G_{1}$ into $S$ phase, with subsequent cell division. Rapamycin leads to failure to downregulate $p 27$, reduced cdk2 and 4 activities, and failure to inactivate $R B$, with subsequent arrest in $G_{0} / G_{1}$. For explanation of abbreviations see text.

transit of the $\mathrm{G}_{1}$ cell cycle restriction point (R point) controlled by the retinoblastoma protein $\mathrm{RB}$ (fig 3).

So how do these mechanistic insights translate into biological effects on VSMCs and/or the reaction to coronary stenting? Firstly, sirolimus is a potent inhibitor of both animal and human VSMC proliferation. Interestingly, high doses of drug are not associated with significant cellular toxicity, an important consideration when using overlapping stents where local drug levels may be increased. Growth arrest is predominantly in $G_{1}$, although post- $G_{1}$ effects may also be important. Rapamycin prevents mitogen induced p27 downregulation in many cells including VSMCs in vitro and in vivo. ${ }^{11}$ In addition, rapamycin resistant cells fail to upregulate p2 $7^{12}$ and p27 knockout cells are relatively resistant (though not wholly so) to rapamycin, arguing that p27 upregulation is a requisite action for rapamycin. In contrast, other studies have found that p27 deficiency does not impair the ability of rapamycin to reduce neointima formation after arterial injury in mice. ${ }^{13}$ Rapamycin induced growth arrest is also associated with reduced cyclin and cdk activity after vessel injury, reduced cyclin D associated kinase activity, and hypophosphorylation of RB. ${ }^{14}$

Secondly, rapamycin reduces VSMC migration, both as isolated cells or migration from explants. This effect may also be caused by p27, as p27 deficient VSMCs are relatively resistant to this rapamycin effect. Thirdly, rapamycin is a potent inhibitor of cell size, a function separable from its inhibition of cell proliferation. Growth of cells to an appropriate size depends upon mTOR and PI-3K dependent signals, and is mediated by S6K1 and eiF4F. Both proteins regulate cell growth by controlling the biosynthesis of translational components that comprise the protein synthetic apparatus, most notably ribosomal proteins. mTOR signalling also maintains levels of expression of surface transporters for not only glucose but also amino acids, low density lipoprotein, and iron, acting as a nutrient sensing mechanism for cell growth.

Fourthly, rapamycin may have significant effects on cell matrix synthesis. For example, rapamycin reduces drug induced pulmonary fibrosis, and hepatic fibrosis in a model of

\section{Abbreviations}

ASPECT: Asian paclitaxel-eluting stent clinical trial cdki: cyclin dependent kinase inhibitor

EEL: and external elastic laminae

ELUTES: European evaluation of paclitaxel eluting stent eiF: eukaryotic initiation factor

IEL: internal elastic laminae

ISS: in-stent stenosis

MLD: minimum luminal diameter

mTOR: mammalian target of rapamycin

PIK: phosphatidylinositol kinase

VSMC: vascular smooth muscle cell

cirrhosis, and extracellular matrix signals that activate S6 kinase are inhibited by rapamycin. As matrix comprises the bulk of the ISS lesion, this effect of rapamycin may be even more important than its antiproliferative effect.

Finally, rapamycin is an inhibitor of inflammation in vessels after injury. Both sirolimus (rapamycin) and tacrolimus inhibit $\mathrm{T}$ lymphocyte proliferation and activation, acting as both anti-inflammatory and immunosuppressant agents. Although it is unclear how important the acute inflammation seen after injury is in vessel wall repair after stenting, rapamycin reduces inflammation "score", an index of the accumulation of inflammatory cells after stenting in pigs. ${ }^{15}$

These biological properties mean that rapamycin eluting stents are potent inhibitors of neointima formation of animal models of ISS ${ }^{15}$ and in humans. ${ }^{23}$ Although catch up is seen at 90 days in pigs, suppression of human neointima formation and low levels of restenosis are seen in humans to 2-3 years.

\section{Paclitaxel}

The taxanes (for example, paclitaxel) are potent antiproliferatives used in cancer. Paclitaxel promotes polymerisation of the $\alpha$ and $\beta$ subunits of tubulin by reversibly and specifically binding the $\beta$ subunit of tubulin, and thus stabilises microtubules. Microtubule disassembly is required for $G_{2}$ transition into $M$ phase; the drugs thus block cell proliferation in $G_{2} / M$. 
In addition, tubulin dimers play an important role in various cell functions, such as maintaining cell and axonal transport in nerve cells. These cellular modifications lead to reduced proliferation, migration, and signal transduction in many cell types. Paclitaxel is highly lipophilic, which promotes a rapid cellular uptake, and has a long lasting effect in VSMCs as a result of the structural alteration of the cytoskeleton. Paclitaxel leads to an almost complete growth inhibition within a dose range of $1.0-10.0 \mathrm{mmol} / \mathrm{l}$, even after a short (20 minute) single dose application, but is cytotoxic (via apoptosis and necrosis) at higher concentrations. These actions, coupled with low solubility, make it a viable candidate for modulating vascular responses to injury and prolonged effects after local delivery.

Although the major action of paclitaxel is on microtubule depolymerisation, important biological processes, such as the activation of some protein kinases and serine protein dephosphorylation, are associated with depolymerisation and are therefore inhibited by paclitaxel. Paclitaxel has also been implicated in activation of a large number of other signal transduction pathways, including induction of cytokines and tumour suppressor genes, indirect cytotoxicity caused by secretion of tumour necrosis factor, vast activation of signal transduction pathways and gene expression, and selective activity against cells lacking functional p53. While these effects occur at much higher concentrations than those seen with systemic paclitaxel treatment, it is possible that paclitaxel eluting stents exert additional effects through these mechanisms on the vessel wall.

Paclitaxel binds to cells in a specific and saturable manner with a single set of high affinity binding sites. Paclitaxel can inhibit vascular smooth muscle cell proliferation in vivo in organ culture or in animal models as a local infusion of native drug or albumen bound nanoparticles or when bound to a stent. ${ }^{16-18}$ In some studies, paclitaxel reduced intimal and medial cell proliferation threefold seven days after stenting and virtually eliminated later intimal thickening. Six months after stenting, neointimal area was twofold lower in paclitaxel releasing stents in some studies, ${ }^{16}{ }^{17}$ although catch up was seen at 90 days in others. ${ }^{18}$

In humans, the use of paclitaxel on drug eluting stents has mostly been positive. Although not yet published, paclitaxel eluting stents dose dependently reduced per cent diameter stenosis in both the ELUTES and ASPECT clinical trials. Similar dose dependent effects were found in TAXUS 1 and 2, although trials of a derivative of paclitaxel, 7-hexanoyltaxol (QP2) eluting polymer stent, revealed minimal intimal hyperplasia at the six month follow up, but with delayed recurrence at 12 months of angiographic restenosis (61\%). Late occlusion leading to non-Q wave myocardial infarction has also been reported after use of paclitaxel eluting stents, caused by stent occlusion. $^{19}$

\section{Potential toxicity and specificity}

Drug eluting stents are local drug delivery vehicles par excellence, resulting in the ability to deliver high local concentrations of drugs whose systemic concentrations would lead to unacceptable side effects, including immunosuppressant and anti-cancer drugs. For example, the conventional toxicity of systemic paclitaxel, including hypersensitivity reactions, cardiotoxicities, neutropenia, peripheral neuropathy, mucositis, gastrointestinal toxicities, alopecia, arthralgias, and myalgias, are not seen with stent implantation. Stent based delivery invariably leads to large concentration gradients, particularly with asymmetrical stent expansion, with drug concentrations ranging from nil to several times the mean tissue concentration over a few micrometres. Characteristics of the delivered drug contribute largely to the delivery efficiency, so that drug concentrations in the arterial wall can be significantly higher for a lipophilic drug such as paclitaxel as compared to a hydrophilic drug. Drug toxicity also depends upon the dose of drug on the stent, the elution profile, the amount of drug bound and retained in the vessel, and blood concentrations. Rapamycin is lipophilic, with good cellular penetration and retention, the latter being dependent in part on $\mathrm{FKBP}_{12}$ expression. In porcine studies, rapamycin is detectable in the bloodstream after stenting, with a peak concentration at one hour, but is undetectable by three days. ${ }^{15}$ At three days, approximately $40 \%$ of drug is still bound to the stent, with tissue concentrations at later time points reported to be similar. ${ }^{15}$ Paclitaxel is also a lipophilic, poorly soluble agent, with good tissue retention characteristics.

This multiplicity of actions of rapamycin and paclitaxel would suggest that they may be ideal agents to inhibit the multiple processes leading to ISS. However, this "nonspecificity" raises the possibility of vessel wall toxicity, and any antiproliferative agent may inhibit division of more than just VSMCs. Indeed, a major concern with both brachytherapy and drug eluting stents relates to failure of complete vessel healing, with subsequent lack of re-endothelialisation and late thrombosis, and medial thinning with stent malapposition. Thus, an ideal agent would exhibit specificity to the vessel intima, without having an effect on endothelial cells or medial VSMCs. Rapamycin does inhibit cell proliferation in both medial and ISS human VSMCs, with a similar potency in vitro (MR Bennett, unpublished observations). However, some specificity for ISS VSMCs may be provided in vivo by the expression of $\mathrm{FKBP}_{12}$. A recent study of differential gene expression in human ISS versus medial VSMCs demonstrated increased expression of $\mathrm{FKBP}_{12}$ in ISS VSMCs. Thus, rapamycin released to the vessel wall may preferentially bind to the intima, the receptor potentially acting as a "sink" and local reservoir for the drug. In porcine studies, rapamycin coated stents did not impair re-endothelialisation and this was complete by 28 days. ${ }^{15}$ However, rapamycin treated vessels had a higher fibrin content, indicating either a delay in arterial repair or delayed degradation of fibrin caused by the drug. Whether this translates into long term deleterious effects of the drug will require follow up of human patients.

Paclitaxel eluting stents also show dose dependent effects on vessel healing. At higher doses, persistent intimal fibrin deposition, intraintimal haemorrhage, and increased intimal and adventitial inflammation are evident, ${ }^{20}$ and in clinical studies high dose paclitaxel has resulted in failure of re-endothelialisation and thinning of the media. Long term treatment with antiplatelet therapy may thus be required to prevent late occlusion. Taxanes can inhibit endothelial cell migration, proliferation, and microtubule formation at systemic doses achieved clinically, which may be beneficial as anti-angiogenesis in atherosclerosis, but may also underlie some incomplete healing. Other tissue responses in paclitaxel treated vessels included incomplete healing, few smooth muscle cells, late persistence of macrophages, and dense fibrin with little collagen.

\section{CONCLUSIONS}

ISS is caused by a number of overlapping biological processes, with different time courses. In human stenting, it is unclear how much each process, such as cell proliferation, resolution 
Theoretical "ideal" properties of an antirestenosis

\section{drug}

- Lipophilic with good tissue retention

- Strong binding/slow elution from polymer

- Antiproliferative without inducing cell death

Potent inhibitor of vascular smooth muscle cells (VSMCs) but not endothelial cells

- Specific to intimal VSMCs

- Antimigration action on VSMCs

- Anti-inflammatory action

- Antiplatelet action

of inflammation, and thrombus reorganisation, contributes to final lesion size. The multiplicity of actions of rapamycin, based upon mTOR acting as a central second messenger, together with favourable kinetics of uptake and retention are likely to underlie its apparent efficacy. Paclitaxel also affects a number of biological processes responsible for ISS. These properties are not unique to rapamycin or paclitaxel, and other drugs with a broad action on multiple biological processes are also likely to be effective. However, the biological profile of these drugs act as a template to judge the likely efficacy of other antirestenotic treatments.

\section{ACKNOWLEDGEMENTS}

MRB is a British Heart Foundation Chairholder.

\section{REFERENCES}

1 Bennett MR, O'Sullivan M. Mechanisms of angioplasty and stent restenosis: implications for design of rational therapy. Pharmacol Ther 2001;91:149-66

2 Sousa JE, Costa MA, Abizaid AC, et al. Sustained suppression of neointimal proliferation by sirolimus-eluting stents: one-year angiographic and intravascular ultrasound follow-up. Circulation 2001;104:2007-11.

3 Morice MC, Serruys PW, Sousa JE, et al. A randomized comparison of a sirolimus-eluting stent with a standard stent for coronary revascularization. N Engl J Med 2002;346:1773-80.

- The RAVEL study - a landmark clinical trial demonstrating a profound suppression of neointima formation and lack of restenosis in patients receiving a sirolimus eluting stent.

4 Moreno PR, Palacios IF, Leon MN, et al. Histopathologic comparison of human coronary in-stent and post-balloon angioplasty restenotic tissue. Am J Cardiol 1999;84:462-6[A9].
5 Farb A, Sangiorgi G, Carter A, et al. Pathology of acute and chronic coronary stenting in humans. Circulation 1999;99:44-52.

6 Yutani C, Ishibashi-Ueda H, Suzuki T, et al. Histologic evidence of foreign body granulation tissue and de novo lesions in patients with coronary stent restenosis. Cardiology 1999;92:171-7.

7 Schwartz RS, Holmes DR, Jr, Topol EJ. The restenosis paradigm revisited: an alternative proposal for cellular mechanisms. J Am Coll Cardiol 1992;20:1284-93.

8 Waller BF, Pinkerton CA, Orr CM, et al. Restenosis 1 to 24 months after clinically successful coronary balloon angioplasty: a necropsy study of 20 patients. J Am Coll Cardiol 1991;17(suppl B):58B-70B

9 Mintz GS, Popma JJ, Pichard AD, et al. Arterial remodeling after coronary angioplasty. A serial intravascular ultrasound study. Circulation 1996:94:35-43.

- Definitive demonstration that remodelling is the major mechanism of restenosis after angioplasty, and neointima formation after stenting.

10 Komatsu R, Ueda M, Naruko T, et al. Neointimal tissue response at sites of coronary stenting in humans: macroscopic, histological, and immunohistochemical analyses. Circulation 1998;98:224-33.

11 Gallo R, Padurean A, Jayaraman T, et al. Inhibition of intimal thickening after balloon angioplasty in porcine coronary arteries by targeting regulators of the cell cycle. Circulation 1999;99:2164-70.

12 Luo Y, Marx SO, Kiyokawa H, et al. Rapamycin resistance tied to defective regulation of p27Kip1. Mol Cell Biol 1996;16:6744-51.

13 Roque M, Reis ED, Cordon-Cardo C, et al. Effect of p27 deficiency and rapamycin on intimal hyperplasia: in vivo and in vitro studies using a p27 knockout mouse model. Lab Invest 2001;81:895-903.

14 Braun-Dullaeus RC, Mann M, Seay U, et al. Cell cycle protein expression in vascular smooth muscle cells in vitro and in vivo is regulated through phosphatidylinositol 3-kinase and mammalian target of rapamycin. Arterioscler Thromb Vasc Biol 2001;21:1152-8.

15 Suzuki T, Kopia G, Hayashi S, et al. Stent-based delivery of sirolimus reduces neointimal formation in a porcine coronary model. Circulation 2001;104:1188-93

- Comparison of dexamethasone and rapamycin eluting stents on porcine vessels, demonstrating dose dependent reduction in neointima formation with both agents, and reduced inflammation at the stent site.

16 Hong MK, Kornowski R, Bramwell O, et al. Paclitaxel-coated Gianturco-Roubin II (GR II) stents reduce neointimal hyperplasia in a porcine coronary in-stent restenosis model. Coron Artery Dis $2001 ; 12: 513-5$

17 Heldman AW, Cheng L, Jenkins GM, et al. Paclitaxel stent coating inhibits neointimal hyperplasia at 4 weeks in a porcine model of coronary restenosis. Circulation 2001;103:2289-95

18 Kolodgie FD, John M, Khurana C, et al. Sustained reduction of in-stent neointimal growth with the use of a novel systemic nanoparticle paclitaxel. Circulation 2002; 106:1 195-8.

19 Liistro F, Stankovic G, Di Mario C, et al. First clinical experience with a paclitaxel derivate-eluting polymer stent system implantation for in-stent restenosis: immediate and long-term clinical and angiographic outcome. Circulation 2002; 105:1883-6.

20 Farb A, Heller PF, Shroff S, et al. Pathological analysis of local delivery of paclitaxel via a polymer-coated stent. Circulation 2001;104:473-9.

- Detailed analysis of the pathology of normal (animal) vessels after stenting with increasing doses of paclitaxel, demonstrating dose dependent toxicity to all components of the vessel wall. 